\title{
Humano, ¿obstinada o sensiblemente humano?
}

\author{
Dominika Partyga
}

$\mathrm{H}$ ace un siglo, poco antes de su muerte, Simmel (1964: 23) formuló la enigmática idea de una sociología filosófica como el 'estudio de los aspectos epistemológicos y metafísicos de la sociedad' que se ocupa de cuestiones que trascienden los límites de la investigación empírica. Inspirándose en la historia intelectual, el proyecto continúa meditaciones de tipo nietzscheanas sobre el destino del individuo moderno en un mundo sin fundamentos seguros, dividido entre, por un lado, el instinto de rebaño para obedecer las exigencias morales de una sociedad decadente, y el proyecto experimental de superación de su propia humanidad, por el otro. A medida que el filosofar modernista se institucionalizaba, y un robusto aparato de herramientas científico-sociales sustituía a la teorización especulativa, no es de extrañar que fuera la 'sociología formal' de Simmel, más que su interpretación de Nietzsche, lo que le asegurara un lugar en el canon sociológico. Más allá de los intentos de resituar la sociología vital de Simmel como parte de su proyecto modernista más amplio (Goodstein, 2017; Kemple, 2018; Pyyhtinen, 2017), el limitado atractivo de su noción de sociología filosófica capta un sentido fundamental en el que la filosofía poskantiana y la teoría social a menudo se encuentran solo para divergir, a pesar de sus vocabularios teóricos compartidos y sus historias disciplinarias entrelazadas. ${ }^{1}$ También apunta a varias vías de pensamiento

\footnotetext{
* Dominika Partyga: London School of Economics.

1 Desde la traducción de Lebensanschauung de Simmel al inglés, los límites entre la dimensión "sociológica" y "filosófica" en el pensamiento de Simmel se han desdibujado de varias maneras productivas (Kemple, 2018; Pyyhtinen, 2017). En esta línea, Goodstein (2017: 250) señala hacia la posición liminar de Simmel entre la filosofía modernista del siglo XIX y la emergente ciencia social moderna como un pensador cuyas contribuciones filosóficas han sido oscurecidas por lo que ella llama 'modos de recepción canonizantes en la sociología’ y una 'comprensión estrechamente analítica de lo que es filosófico'. Me gustaría
} 
tentadoras, aunque peligrosas, que se abren al recuperar esas conexiones, como el proyecto de filosofía antropológica de Gehlen, que proporcionó una base científico-social para poner a prueba algunas de las afirmaciones naturalistas más controvertidas de Nietzsche, ofreciendo una conexión entre los registros científico y filosófico para elevar a la humanidad más allá del sistema de valores del decadente 'animal de rebaño' (Schacht, 2015).

En Debating Humanity, Daniel Chernilo (2017) pretende tender puentes conceptuales entre esos campos, reconstruyendo la idea de la sociología filosófica como un reencuentro con las preguntas "incontestables" que uno podría asociar en un primer momento con la tradición filosófica. En particular, con la corriente poskantiana de la antropología filosófica que considera al 'hombre' como una especie que se cuestiona sobre las condiciones de sus propias prácticas de creación de significado. Aquí no se sigue a Nietzsche ni a Simmel. Chernilo intepreta el proyecto original de Simmel como orientado a la 'aclaración epistémica' (15). Sin embargo, la idea más amplia de lo humano como fuente de valor encuentra su eco en la afirmación de Chernilo sobre la centralidad de esta figura para entender la vida social, tanto en un sentido epistémico como moral. Partiendo de una interesante caracterización de los seres humanos como 'árbitros últimos de lo que está bien y lo que está mal' (1), Chernilo pretende sacar a la luz las apuestas normativas de la sociología y reconstruir su orientación humanista implícita. Podría decirse que es esto último lo que hace que su proyecto sea provocador en el panorama teórico contemporáneo: la resignificación de la sociología filosófica en el siglo XXI se enmarca en el predominio de temas ampliamente foucaultianos y posthumanistas en el pensamiento científicosocial, que enfatizan los legados violentos y los límites excluyentes de 
conceptos como 'hombre' tal y como se construye a través de las nociones de diferencia del otro (mujer/esclavo/negro/animal), aunque a menudo recurren a lo que Chernilo llama motivos normativos 'tradicionales' como la libertad o la igualdad.

Tomando en cuenta el carácter amplio y ambicioso del proyecto, sería imposible pasar revista a la multitud de ejes metodológicos y sustantivos que transpiran en este libro. En mi respuesta, me gustaría centrarme en dos aspectos de la sociología filosófica que resuenan con mi interés en las implicaciones del pensamiento humanista crítico de Nietzsche para la teoría social. En primer lugar, la crítica de Chernilo a la falsa orientación 'anormativa' de la sociología y, en segundo lugar, su propuesta de un principio universalista de humanidad. Por mucho que comparta los sentimientos de Chernilo por una sociología que brinde vida a cuestiones normativas complejas, una postura que departa más allá del binarismo 'humanismo versus anti-humanismo' me parece más productiva en este contexto, especialmente a la luz de los espectros del colonialismo que aún acechan a las complejas historias de los humanismos. Antes de hablar de lo que está en juego aquí a nivel político, quisiera revisar las transiciones clave de Chernilo al navegar por el paisaje intelectual de la posguerra.

Como sugiere el título, el libro se perfila como un debate. En el primer capítulo, Chernilo define los términos dentro del espectro de la apropiación de Sartre del humanismo y el rechazo de Heidegger a sus presupuestos antropocéntricos. Más precisamente, la interrogante heideggeriana acerca de si el daño causado por el humanismo no es suficientemente obvio. Tras pasar revista a diversos aspectos de la ola antihumanista de posguerra que ha provocado dicha pregunta, Chernilo examina detenidamente la crítica de Heidegger a la tradición metafísica occidental para, en última instancia, argumentar contra su 'elitismo a ultranza' (47), empleando 
una estrategia interpretativa clásica basada en antitesis y refutaciones. De hecho, las connotaciones específicas de la acusación de Chernilo se ponen de manifiesto en el contraste que establece entre la retirada de Heidegger a los ámbitos 'impolutos' del ser y la poesía, y la concepción existencialista de Sartre de la libertad concebida como 'el ser del hombre' (32). Para Chernilo, la posición de Sartre no está exenta de defectos en la medida en que está ligada a un sentido inflado del dominio humano, pero su 'preocupación por la libertad, la dignidad y la igualdad humanas' (61) proporciona una base más igualitaria para delinear los términos clave de la sociología filosófica. La elección de Chernilo de pensadores que representan los dos polos del debate: Arendt, Parsons, Jonas, Habermas, Archer y Boltanski en el campo kantiano/sartriano - frente a Taylor en el lado 'anti-humanista' de Heidegger refleja esta tensión central que anima el libro.

En los capítulos restantes, Chernilo rastrea las preconcepciones de lo humano que sustentan las obras de dichos autores para reconstruir, en última instancia, un principio universalista de humanidad construido en torno a la idea de nuestras 'propiedades antropológicas' compartidas. Es importante destacar que esta idea se entiende no como una esencia singular o una propiedad central que marcaría el límite cognitivo de lo humano, sino en términos de capacidades relacionales que son 'autónomas frente a la sociedad, aunque su actualización sea en sí misma social' (229). Se trata de un proyecto complejo tanto en su contenido como en su concepción, pero el proceder metodológico de Chernilo -un rasgo antropológico por cada pensador- le permite presentar el argumento principal de una forma característicamente lúcida, al tiempo que plantea diferentes conjuntos de cuestiones en siete capítulos ordenados cronológicamente: 1) autotrascendencia (Arendt), 2) adaptación (Parsons), 3) responsabilidad (Jonas), 4) lenguaje (Habermas), 5) evaluaciones fuertes (Taylor), 6) reflexividad (Archer) y 7) reproducción de la vida (Boltanski). La concepción 
emergente de la humanidad pretende aclarar los fundamentos sobre los que los científicos sociales podrían elaborar argumentos normativos sobre las instituciones y las prácticas que habitamos. En este punto, se vislumbra un eco de la distinción post-nietzscheana de Simmel entre los puntos de vista de la humanidad y la sociedad, aunque con dos diferencias fundamentales realizadas mediante una operación única. Chernilo articula una antropología filosófica positiva orientada en torno a universales, que luego se traducen en principios normativos estáticos. Si se llevara la sociología filosófica de Chernilo a su extremo, quizá podría leerse como un marco operativo en el que esta distinción humanidad/sociedad se moraliza, en la que el papel del sociólogo se acerca peligrosamente al de un juez que evalúa hasta qué punto las prácticas sociales 'promueven o incluso socavan el desarrollo de nuestros potenciales humanos genéricos' (230).

En cuanto al potencial de esta visión, resulta difícil encontrar opositores al proyecto de establecer puentes entre 'los aspectos orgánicos e intelectuales de nuestra humanidad' (233) si esto se entiende como la idea de que las variaciones socioculturales, los contextos políticos y las (ir) regularidades orgánicas se entrelazan en formas terriblemente complicadas, y que podemos aprender mucho sobre las sociedades estudiando este punto de encuentro -aunque Parsons pueda parecer una elección sorprendente para teorizar dicha complejidad. Por otro lado, los alcances normativos de la sociología filosófica pueden llegar a ser como más controversiales. A pesar de todos los vínculos que se puedan establecer entre (hetero) normatividad y violencia epistémica, Chernilo pretende reencantar lo normativo convenciéndonos de que está ahí, nos guste o no. La sociología, con su 'restricción normativa auto-impuesta' (215), argumenta el autor, nos engaña al hacernos creer que es posible describir construcciones de normas y procesos de normalización sobre una base puramente científica y empírica. Sin embargo, el problema de lo normativo resuena al de lo 
humano: suele abrirse paso por la puerta de atrás, porque los estudios científico-sociales se realizan en última instancia desde una perspectiva humana, si no demasiado humana, es decir, desde una perspectiva de los sujetos de habla, de un 'nosotros' que se preocupa. Chernilo realiza movimientos críticos contra Latour (13) y Braidotti (15) en esta línea, pero es en última instancia su análisis de la 'antropología vacua' de Bourdieu lo que le lleva a argumentar que 'la sociología contemporánea dominante no parece haber aprendido las lecciones correctas' (9) sobre la importancia de conceptualizar la normatividad sociológicamente. Un ejemplo sería la 'noción reduccionista de los intereses propios' de Bourdieu, aunada a 'una concepción igualmente reduccionista de lo social como un espacio de lucha constante' (10). Para Chernilo, esta comprensión de lo normativo en términos de posiciones socio-materiales transfiguradas es emblemática de la tendencia más amplia de la sociología hacia la descripción de la vida social como 'anormativa' (10) - una tendencia que el proyecto de la sociología filosófica pretende desafiar.

Por supuesto, esta es una burda simplificación de los sutiles argumentos de Chernilo sobre la construcción del homo sociologicus, pero llega al núcleo de por qué la sociología filosófica prescinde del mito weberiano de la neutralidad axiológica: 'si las justificaciones son fundamentales para lo que hacen los actores ordinarios en su propio mundo de la vida y la sociología no tiene ningún privilegio epistémico, ¿por qué los sociólogos no deberían elaborar argumentos normativos?' (227). En el momento en que Chernilo formula esta pregunta, a más de 200 páginas del libro, es tentador leerla como retórica. Esta surge en el contexto de su interpretación de la distinción de Boltanski entre los aspectos pre-sociales de las capacidades de juicio de los actores frente a los factores sociales. Aunque Chernilo espera retener esta orientación básica, sigue siendo fiel al espíritu de las refutaciones que demuestra en los otros capítulos, y critica a 
Boltanski por su 'tibio' (224) compromiso durkheimiano de separar hechos y normas frente al peso relativo de la evidencia empírica en La Condición Fetal. En la interpretación de Chernilo, el estudio de Boltanski sobre el aborto ‘se inclina hacia su rechazo normativo' (266) en la medida en que sus descripciones de los regímenes reproductivos de la violencia parecen llevar un matiz moralizador. En este sentido, Boltanski no cumple con la tarea que Chernilo asocia al papel de un juez: llegar a una conclusión explícitamente normativa que 'tomará partido' (225) tras un juicio sociológico imparcial.

La distinción entre los registros descriptivo y normativo es crucial en este punto. Además de tratarlos como distintos a nivel analítico, Chernilo insta a los sociólogos a teorizar su interacción. La idea de 'descripciones normativas' (230) pretende abordar este fracaso de la imaginación sociológica. La forma en que Arendt condena los campos de concentración presenta, desde su punto de vista, una de las formas más agudas de descripciones normativas: la cuestión es que sería difícil describirlos de otra forma que no fuera como una amenaza a nuestra humanidad compartida. Queda por ver cómo podría funcionar esto en escenarios en que la cuestión de vida y muerte no sean tan explícitos, pero el atractivo que Chernilo encuentra en esta idea refleja un hábito intelectual más amplio que parece ser omnipresente en el campo 'humanista'. Al igual que Archer defendió la dicotomía estructura-agencia como la más humana de las experiencias cotidianas, y Parsons tomó la salud como un valor universal, Chernilo da a entender que hay algo noble, o al menos intuitivo, en el impulso de reflexionar en primer lugar sobre la 'especificidad y valía' humana (234). Esto hace que el proyecto sea normativo por diseño: si preguntas sobre 'Dios, libertad y mortalidad' (234) siguen persiguiéndonos a la mayoría de nosotros, la sociología filosófica convierte esta insatisfacción distintivamente humana en un programa. Pues, debatir el sentido de la vida se convierte en 
una fuente de virtud en sí misma, en lugar de algo que podríamos desear historizar como una adicción ambivalente del animal humano.

Algunos podrían verse tentados a concluir que el proyecto de Chernilo se basa en una comprensión circular de la normatividad, en la que las condiciones siempre en retirada bajo las cuales los afectos, valores y compromisos humanos podrían adquirir fuerza normativa se establecen fijamente como estándares externos. Otros objetarán la idea subyacente de que existe una especie de 'nosotros' unificado -en este caso nosotros, los sociólogos filosóficos- cuyas deliberaciones sobre los 'potenciales humanos genéricos' pueden ser la base para reconstruir ideas normativas irreductibles desde cero. Esta pregunta clásica sobre la pretensión y posibilidad de universalidad de hablar en nombre de la humanidad se encuentra en el centro del debate entre Sartre, Heidegger y Derrida que enmarca el proyecto de Chernilo. Es menester volver a algunos movimientos clave que Chernilo hace en este contexto. Esto ayudará a aclarar por qué la comprensión de Chernilo de lo normativo como una cuestión de juicio moral está en tensión con una noción más inmanente de la crítica que podríamos leer en la trayectoria nietzscheana-foucaultiana, en la que las reconstrucciones de las normas que subyacen a la realidad social están sujetas a la crítica 'genealógica' (Han-Pile, 2016). En este caso, las normas serían vulnerables y contingentes $-y$, por tanto, estarían sujetas a pruebas en contextos particulares- en lugar de ser universalizables en sistemas morales estáticos.

En la interpretación de Chernilo, es la distinción post-kantiana entre lo particular y lo universal la que constituye la base sobre la que Sartre argumenta la relación dialéctica entre la responsabilidad, la libertad individual y la libertad de la especie humana. Un célebre ejemplo de El Ser y la Nada ayuda a corroborar este punto. Para Sartre (1957), incluso un prisionero de guerra es 'éticamente' libre porque puede elegir 
cómo reaccionar ante su encarcelamiento y, al hacerlo, compromete su humanidad con la desesperación o la resistencia. El sociólogo filosófico presumiblemente contraargumentaría que este prisionero no tiene pleno control de su contexto y circunstancias socioculturales, y mucho menos es dueño del universo, pero en última instancia está de acuerdo con Sartre en que nuestra capacidad de ser responsables es autónoma frente a la sociedad, y no mediada socialmente (por ejemplo, por consideraciones de género o raciales). En consecuencia, se supone que no debemos pensar en este prisionero simplemente desde una perspectiva ANT/estructuralista como producido por las bases de datos de ADN racializadas, las exposiciones desiguales a la contaminación del aire y las prácticas policiales militarizadas, sino formular explícitamente una evaluación normativa de las prácticas e instituciones que disminuyen su florecimiento. La evaluación normativa sigue directamente del juicio sociológico.

Con este tipo de movimiento, hemos viajado a través de los contextos y los tiempos de una manera verdaderamente universalista, pero el punto de Chernilo es, en cierta medida, precisamente sobre el poder de la abstracción: es la clave para entender por qué las dos formas de pensar en este prisionero no son dos caras de la misma moneda. En la medida en que la propuesta de Chernilo se enmarca en contra de concepciones particularistas de raza, género, clase y sexualidad, tiene importantes implicaciones metodológicas y políticas. Diferencias reproducidas socio-molecularmente -que hacen que sea más probable que un joven negro acabe en esa prisión- se disuelven en una llamada a lo común: deberíamos utilizar 'la corporeidad como un recurso que también trabaja para su trascendencia' (198), en términos que Chernilo hace eco de Archer. Este enfoque en la corporeidad es inmensamente importante para interrogar cómo la diferencia cultural se convierte en diferencia corporal. Sin embargo, la orientación universalista podría ocultar la importancia sociológica de comprender por qué, cómo y 
dónde sucede que diferencias particulares han adquirido una importancia jerárquica persistente, cómo estas se reproducen en el tejido social y, lo que es más importante, cuál es su relación con las pretensiones de saber y conocimiento.

Esto plantea la cuestión más amplia, en la que Chernilo busca intervenir, de la relación entre el conocimiento sociológico y lo público. La sociología filosófica es más prometedora como una sociología pública en lugar de un debate que se lleva a cabo en una torre de marfil, pero las conexiones entre los registros políticos y académicos de la producción de conocimiento podrían trazarse más explícitamente. En The Politics of the Human, Phillips (2015) advierte contra la confusión de los dos, argumentando que la mayoría de las apelaciones idealistas a nuestra humanidad compartida vacían el cuerpo de su género, raza y sexualidad en formas que aseguran las relaciones normalizadas de poder y privilegio. En principio, trascender las particularidades humanas parece una idea sensata, pero se basa en una resbaladiza distinción entre "un núcleo abstracto y una diferencia noesencial' (Phillips, 2015: 12). Como nos recuerda Phillips, es esta última lo que impulsa a los individuos a afirmar su humanidad en contextos políticos, lo que se manifiesta en las luchas por reformular los derechos de las mujeres como derechos humanos. La sociología filosófica de Chernilo, sin duda, apenas se preocupa por el estatus 'cognitivo' de esas reivindicaciones, pero el vínculo entre los marcadores socioculturales y lo que Chernilo llama 'arraigamientos' (21) no está del todo claro. El cuerpo masculino blanco se convierte en el estándar invisible con el que se mide 'todo lo demás'. Al reflexionar sobre cómo las posiciones autorizadas para hablar se hacen más fácilmente disponibles precisamente para esos cuerpos en varios espacios, desde el parlamento británico hasta el mundo académico, Puwar (2004: 76) describió esto como una 'universalidad vacía de la blancura sobre la que se promulga la posición de hablar por todos'. Esto nos recuerda que los 
cuerpos de conocimiento (textos) en los que debatimos sobre la humanidad son también espacios sociales, estructurados a través de políticas e historias particulares.

En última instancia, si uno está dispuesto a adherirse a la visión de Chernilo podría entenderse entonces como una cuestión nietzscheana sobre el "estilo" y las gramáticas de la escritura (filosófica), planteada por Derrida (1998) en Los Fines del Hombre. Chernilo revisa esta obra en el capítulo inicial centrándose en rebatir la lectura que Derrida hace de Heidegger, pero también admite que algunos aspectos de sus interpretaciones pueden parecer 'controvertidos o incluso parciales' (21). Permítanme entonces seguir la lectura de Shrift (1988), ya que revela las formas en que Derrida 'utiliza' figuras nietzscheanas como paradigma de pluralidad, multiplicidad y juego, que frustra el anhelo logocéntrico de elegir entre dos alternativas para responder a la pregunta antropológica ‘¿quiénes, nosotros?’ -en este caso, humanistas versus anti-humanistas. En esta trayectoria, Fines del Hombre puede leerse como una invitación a deconstruir la forma (el estilo) que podría adoptar cualquier respuesta a este binario como íntimamente ligada a la sustancia (el contenido) del debate, planteando preguntas sobre las implicaciones del sujeto escritor en redes textuales preexistentes en las que los signos ya llevan huellas de la posición de sujeto disponibles para el autor. ${ }^{2}$ La cuestión del estilo apunta a la tensión entre los términos hegemónicos en los que se abordan algunos temas - especialmente cuando se abordan temas 'universales', como la humanidad - y el interlocutor

\footnotetext{
Esta 'cuestión de estilo' nietzscheana está más pronunciada en Spurs: La cuestión del estilo, donde Derrida (1979) explora el estilo plural y antidialéctico de Nietzsche vinculándolo al tropo polifacético de verdad/mujer/castración en los escritos de Nietzsche. Leído como una intervención en el feminismo de la segunda ola de los años 70 , en particular las deconstrucciones de la concepción metafísica y esencialista de la mujer, este texto sigue provocando diversas controversias en relación con la cuestión de lo que significa escribir desde la posición de una minoría (por ejemplo, la mujer), y los peligros que implica la consideración de la categoría política de 'mujer' como un tropo retórico, que podría decirse que oscurece sus realidades socio-materiales (véase Verkerk, 2017).
} 
marcado por el género y la raza que se dirige a lo particular. Cuando se escribe sobre temas filosóficos autorizados, es difícil no convertirse en 'un clon de la norma de habla/somática del hombre blanco en el mundo académico' (Puwar, 2004: 73): escribir desde la minoría es arriesgarse a la ininteligibilidad. La tensión entre lo particular y lo universal se reifica en y a través del estilo.

Visto a través de esta lente lúdica, la respuesta de Chernilo a Sartre, Heidegger y Derrida parece tener algo en común con el estilo del debate británico: se asemeja a un concurso de género con dos equipos, varias rondas y un jurado que a menudo se inclina hacia una de las posiciones asignadas. Chernilo, sin duda, defiende su bando sartriano con mucha gracia, pero cabe preguntarse si los resultados hubieran sido los mismos si hubiera enmarcado el libro en términos más heideggeranos, o si hubiera experimentado más allá de las oposiciones binarias. Tal vez resultaría que los antihumanistas hace tiempo que no existen, y que las acusaciones de criptonormatividad de Taylor y Habermas contra Foucault (266-267) oscurecen formas más sutiles de conceptualizar lo normativo. En la medida en que las problematizaciones históricas de Foucault oscilan entre los registros descriptivo y normativo, su concepción de la crítica como una práctica relacional del yo podría ofrecer caminos para pensar la normatividad sobre bases menos deliberativas y deontológicas (véase Han-Pile, 2016). En esta trayectoria, las indagaciones de Butler (2016) sobre las condiciones éticas y los marcos epistémicos en los cuales ciertos sujetos se hacen diferentemente 'dolientes' complicarían la apelación idealista de Chernilo a la 'empatía universal' (33), ya que los vínculos afectivos asimétricos producidos a través del duelo y el luto revelan cuánto de esta vulnerabilidad compartida permanece negada. También podría apuntar hacia varias corrientes radicales del humanismo no binario que pretenden rescatar el corroído proyecto occidental de sus más crueles abusos deshumanizadores, desde el 
famoso diálogo de Fanon con Sartre sobre los apegos coloniales ‘demasiadohumanistas' a la jerarquía racial, hasta el humanismo planetario de Gilroy (2014) delineado a través de una crítica explícita y sistemática del racismo.

Dejando a un lado mis prejuicios contra los binarios, las refutaciones y los debates, no se trata de cuestionar la promesa de una discusión sociológica sobre la normatividad, sino, por el contrario, de afirmar sus desafíos. Puede que la sociología reciba la versión anglófona de lo humano que se merece, con un toque cosmopolita. No es un modelo ni un espectro, sino un punto de vista desde el que los sociólogos estudian la interacción de la sociedad, los individuos, la cultura y la naturaleza, pero que rara vez se atreven a interrogar. Una vez que dirigimos nuestra mirada hacia ese punto ciego, también es importante recordar que esta mirada, al igual que la práctica de la construcción de teorías abstractas, no ve 'desde ninguna parte'. Así pues, si hay una provocadora lección machista-masculina de Nietzsche que se me permite extraer: 'mira lo suficiente hacia el abismo, el abismo también mira hacia ti' (Nietzsche, 2003, \146). A pesar de todos sus defectos al formular la noción de 'sociología filosófica' cómplice con una óptica europea de hombres blancos, Simmel (1964: 25) parece haber reconocido precisamente esto cuando advirtió que la sociología filosófica depende más de 'las visiones del mundo individuales y partisanas' que de las ramas empíricas de la investigación sociológica. Quizás el significado de esto sintonice con algo más que una nota al pie de página sobre el papel de la posicionalidad en los ejercicios abstractos de construcción teórica: en un sentido genealógico, son las historias de los conceptos, los dispositivos narrativos y las prácticas retóricas a través de las cuales contamos historias sobre el animal humano adicto al significado las que necesitan ser cuestionadas, ya que no hay un punto de vista puramente técnico y neutral desde el que interrogar a la humanidad. 


\section{Referencias}

Butler, J. (2016) Frames of War: When is Life Grievable?. London: Verso.

Derrida, J. (1979) Spurs: Nietzsche's Styles. Chicago: University of Chicago Press.

Derrida, J. (1998) Los Fines del Hombre. Márgenes de la filosofía, Madrid: Cátedra.

Gilroy, P. (2014) Humanities and a New Humanism. The Tanner Lectures on Human Values. New Haven: Yale University.

Goodstein, E. (2017) Georg Simmel and the Disciplinary Imaginary. Stanford: Stanford University Press.

Han-Pile, B. (2016) Foucault, normativity and critique as a practice of the self. Continental Philosophy Review, 49(1), 85-101.

Kemple, T. (2018) Simmel. Medford, MA: Polity Press.

Nietzsche, F. (2003) Beyond Good and Evil. London: Penguin Books.

Partyga, D. (2016) Simmel's reading of Nietsche: the promise of "philosophical sociology” · Journal of Classical Sociology. 16(4), 414-437.

Phillips, A. (2015) The Politics of the Human (The Seeley Lectures). Cambridge: Cambridge University Press.

Puwar, N. (2004) Space invaders. Race, Gender and Bodies Out of Place. London: Berg Publishers.

Pyyhtinen O (2017) The Simmelian Legacy. Basingstoke: Palgrave Macmillan.

Sartre, J.P. (2004) El ser y la nada. Buenos Aires: Losada.

Schacht R. (2015) Gehlen, Nietzsche, and the Project of a Philosophical Anthropology. In: Honenberger P. (ed) Naturalism and Philosophical Anthropology. London: Palgrave Macmillan.

Schrift, A. (1988) Foucault and Derrida on Nietzsche and the End(s) of Man. In: Krell DF and Wood D (eds.), Exceedingly Nietzsche: Aspects of Contemporary Nietzsche Interpretation (pp. 131-149). London: Routledge.

Simmel, G. (1964) Fundamental Problems of Sociology (Individual and Society). In: Wolff, KH (Ed.), The Sociology of Georg Simmel (pp. 3-84). New York: The Free Press.

Verkerk W. (2017) Transgendering Nietzsche: Male Mothers and Phallic Women in Derrida's Spurs. PhiloSOPHIA, 7(1), 99-108. 